\section{THE TREATMENT OF EXCESSIVE} HAMORRHAGE :

SALINE INTRAVENOUS INJECTION OR TRANSFUSION VंERSUS INJECTION OF SALINE FLUID INTO THE AXILLARY OR OTHER CELLULAR TISSUE.

By CHARLES E. JENNINGS, M.D., M.S., F.R.C.S., Formerly Assistant-Surgeon North-West London Hospital.

THE case reported by Mr. Arthur H. Dodd, ${ }^{1}$ as well as others which have previously been published, show that the injection of a pint, more or less, of saline fluid into the subcutaneous cellular tissue is one of the methods by which the

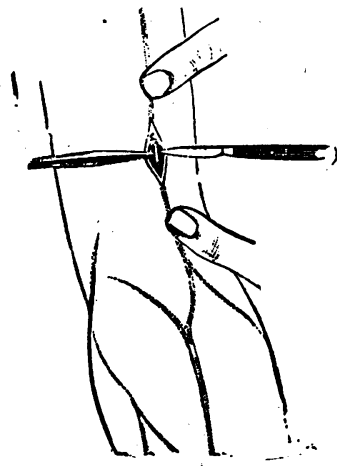

Fig. r.

effect of otherwise fatal hæmorrhages may be counteracted but Mr. Dodd's case also shows that the absorption of the fluid from the cellular space is tardy; " in about two hours sideration, as the patient may easily die before absorption from the subcutaneous tissue has been obtained, and I should say that there are many extreme cases in which intracellular injection would altogether fail, but in which intravenous

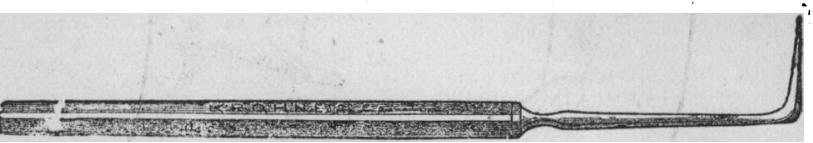

Fig. 2.

injection would certainly succeed. These reasons must form my apology for again calling attention to the instruments for saline intravenous injection and for transfusion of blood, invented by myself some years ago, and of which a complete

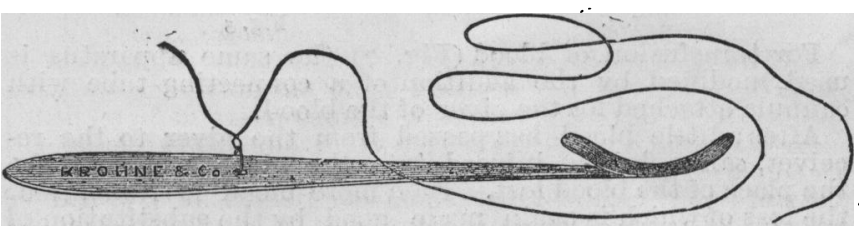

Fig. 4.

description will be found in my published work on the subject. My method aims at precision and certainty. The accompanying illustrations demonstrate the steps of the operation :

Fig. I shows a vein exposed and its sheaf being opened by the use of dissecting forceps and.scalpel.

Fig. 2 shows a phlebotome by means of which the anterior

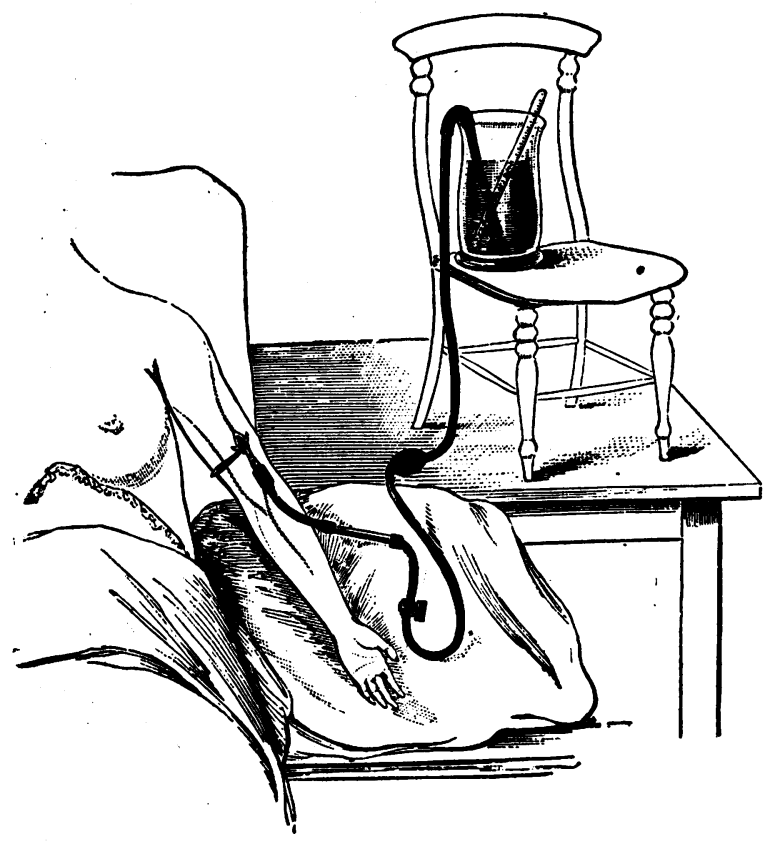

Fig. 6.

there were indications of slight improvement, and a very feeble pulse was detectable at the radials."

Mr. Dodd had injected "a pint of saline fluid into the axillary tissue," now had he injected the same quantity of fluid, or perhaps less, into a vein, the beneficial result would have been almost instantaneous instead of having been postponed for two hours.

In an extreme case, of course, time is of the utmost con1 BRITISH MEDICAL JOURNAI, January 25th, 1896, p. 209 7,

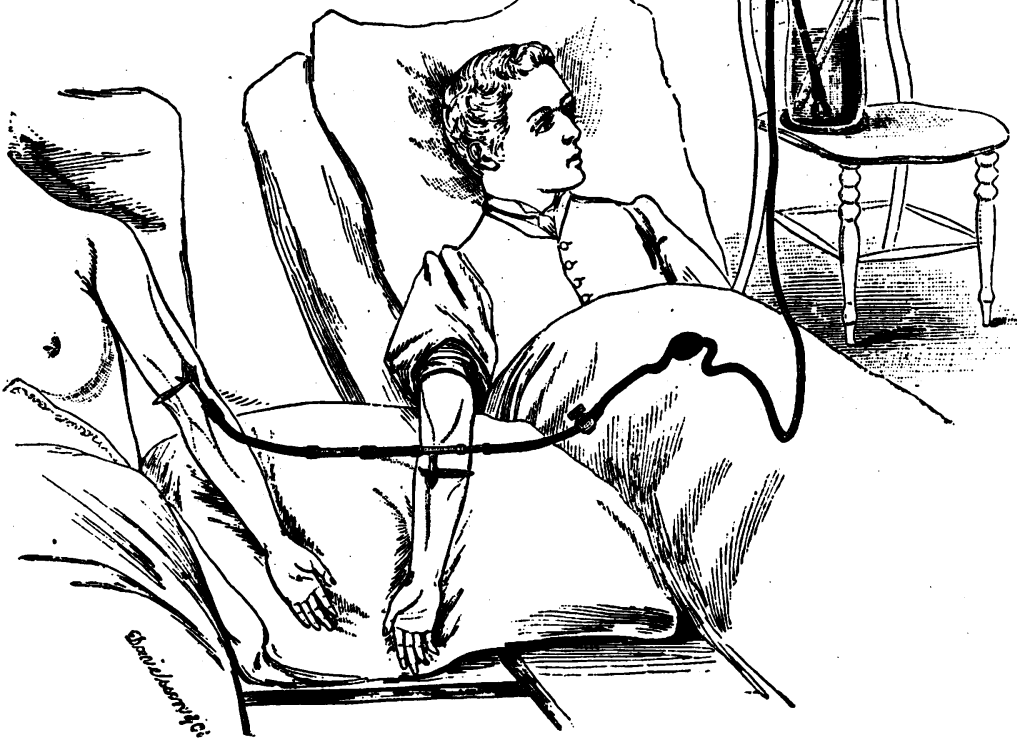

Fig. 7

wall of the vein can be opened and the tip of the cannula guided into it, as shown in Fig. 3 .

Fig. 4 shows a cannula pin, armed with a waxed silk ligature. After the cannula has been passed into the vein, the point of the cannula pin is passed beneath the vein. The cannula is then securely lashed into its position within the vein, as shown in Fig. 5.

Fig. 6 shows the complete apparatus working, and the saline fluid flowing into the vein. 


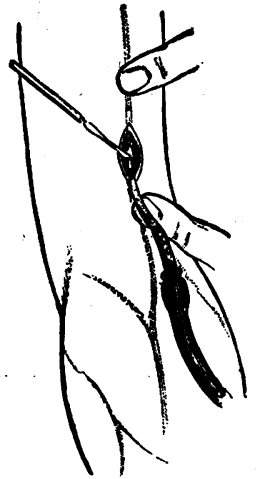

Fig. 3.

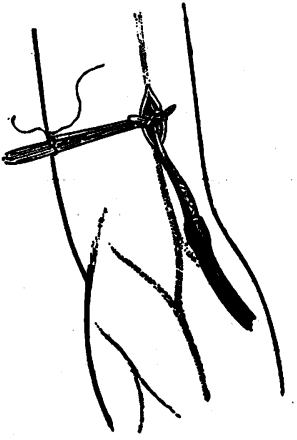

Fig. 5
For transfusion of blood (Fig. 7) the same apparatus is used, modified by the addition of a connecting tube with cannula attached for the giver of the blood.

After a little blood has passed from the giver to the receiver, saline fluid is infused into the giver's veins to take the place of the blood lost. Then more blood is transfused the loss of which is again made good by the substitution of saline fluid. By this plan the giver scarcely misses the loss of some 14 or 16 ounces of blood, and does not faint during the operation.

\section{A CASE OF BULLET WOUND OF THE PREGNANT UTERUS.}

By ARTHUR W. PRICHARD, M.R.C.S., Senior Surgeon to the Bristol Royal Infirmary.

THE following case, in my opinion, will be of interest. It is the only case that I can find recorded of a bullet penetrating the pregnant uterus and intestines, and though it ended fatally, yet for nearly a week it gave us hopes that the patient would escape with her life. The facts are these :

On the morming of Thursday, January 23rd, the patient, Mrs. W., aged 28, who was about seven months advanced in pregnancy, awoke at 7.30, kissed her husband, and smoothed his face with her hand, but did not speak to him. He got out of bed, and immediately shot her with a revolver in the abdomen. She rushed from the room, and as she did so she heard and saw another shot, by which her husband killed himself.

She was admitted into the Bristol Royal Infirmary about Io A.M. in a collapsed condition, and the usual restoratives were at once applied. I saw her at I P.M. There was then considerable reaction, and she was able to answer questions as to the occurrence. The entrance wound of the bullet was 3 inches above and 2 inches in front of the right anterior superior spine; there was little sign of hæmorrhage, and no great resonant distension of the abdomen. The fœtal heart was easily heard. A probe introduced would not pass through the muscles of the parietes, and as there was no distinct evidence that the bullet had penetrated the abdominal cavity 1 resolved to wait a few hours before operation.

About 6 P.M. the patient's pulse was quickening, distension was increasing, and $\mathrm{Mr}$. Thomas Carwardine, the house surgeon, to whom I am greatly indebted for the unremitting care which he gave to the patient throughout the case, found a bruised spot under the skin on the opposite side of the body, half-an-inch above and 5 inches behind the left anterior superior spine, and extracted the bullet under cocaine. He then summoned a consultation.

At 8.30 I made a median incision of the parietes from the umbilicus to 2 inches above the pubes, and could easily see the entrance and exit wounds on the sides of the uterus. I had to prolong the incision 2 inches higher to enable me to put my hand round the uterus, and, having put on a temporary rope écraseur, performed Cæsarean section. The dead fœtus and placenta, both shot through, were removed without hæmorrhage; and, seeing the danger of leaving a uterus with two holes in it in the abdomen, I substituted a wire clamp for the rope ecraseur, and removed the uterus I inch above it, fixing the pedicle outside the abdomen at the lower part of the wound by hysterectomy pins. So far the operation had not lasted long. On examining the intestines I found three coils of small intestine perforated, the wounds of entrance and exit being markedly dissimilar. I closed each of these six apertures by a "purse string" silk suture, Mr. Carwardine tucking in the protruding mucous membrane in the exit wounds as I tied the knot. An artery in the mesentery that was shot through and bleeding was ligatured, and then I emptied the peritoneal cavity of blood and clot, having carefully washed every part with boracic lotion. A big glass drainage tube with a gauze drain in it was put in Douglas's pouch, and I sutured and dressed the abdominal wound, leaving the drainage tube next to the pedicle. The operation took one hour and a-half.

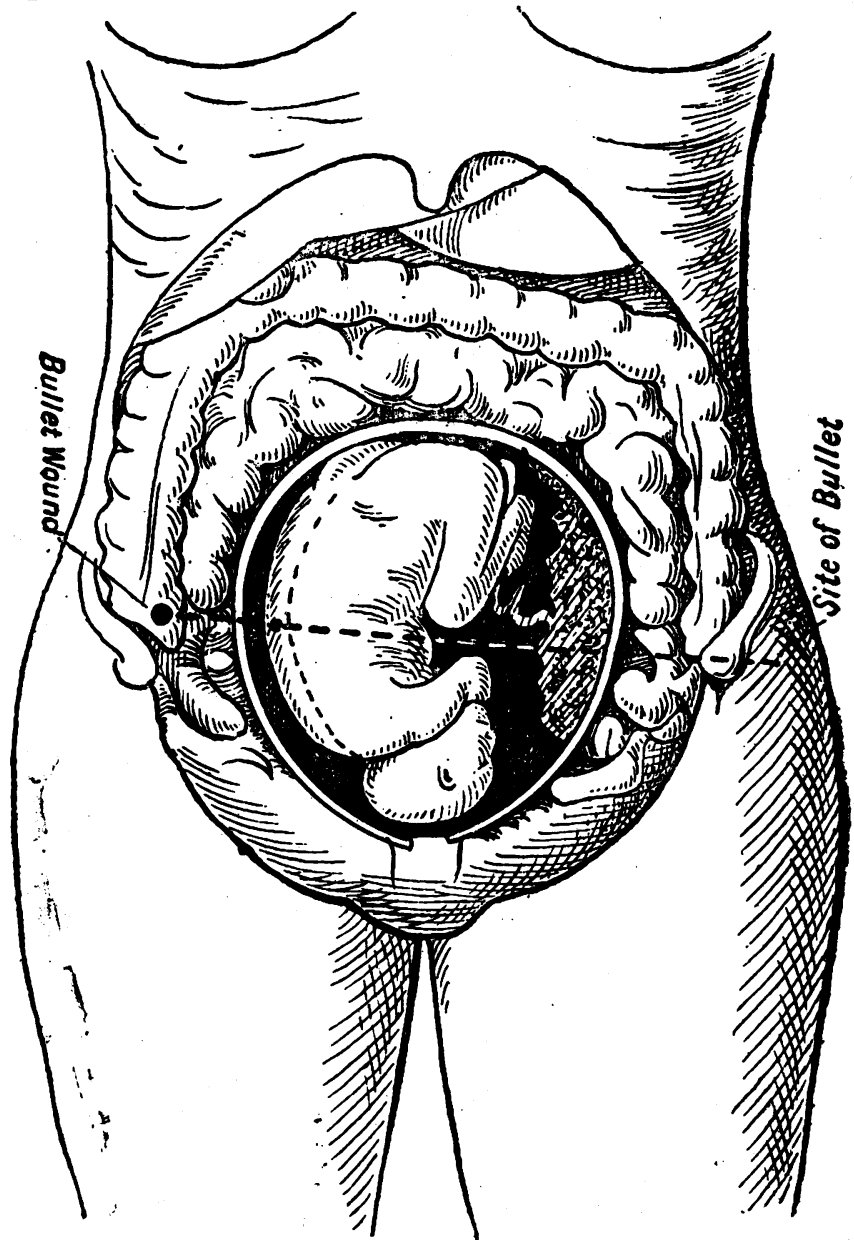

Everything seemed to be going fairly well for the next three or four days. There was some vomiting, but little abdominal distension. She was fed per rectum entirely for two days, and a turpentine enema on the second day, repeated severa times afterwards, relieved the bowels of flatus. A natural stool without any sign of blood was passed on the fourth day, and this was followed by slight diarrhœa. A brownish vaginal discharge came on. She did not complain of great pain, and when it came on $\frac{1}{3}$ gr. of morphine hypodermically gave her several hours' sleep. After the fourth day she took stimulants-brandy and champagne, thin gruel, Brand's essence of peptonised cocoa by the mouth, and until the afternoon before her death I had reason to hope for her recovery. The glass drainage tube was pumped out two or three times a day, and sanguineous fluid, which became purulent later, to the amount of 3 or 4 drachms each dressing, was drawn out. The pedicle was kept dusted with boracic powder. 